\title{
Farrow, Siegel, and Stacy Plan 1989 MRS Spring Meeting in San Diego
}

Robin Farrow, Richard Siegel, and Angelica Stacy are making plans for the symposia to be held at the 1989 MRS Spring Meeting in San Diego, California, April 2428 at the Town and Country Hotel. According to Farrow, "The meeting will feature symposia on topics of continuing interest together with a variety of new topics reflecting recent exciting advances in materials science." New topics include materials for optical storage of information, ultrathin magnetic films and multilayers, bioemulated materials, space-compatible materials, semiconductor clusters, and low temperature synthesis of materials. Symposia on compound semiconductor heterostructures, rapid and integrated semiconductor processing, ion beam processing, and the structural chemistry of high temperature superconductors are also planned.

Look for more details about the 1989 MRS Spring Meeting in San Diego in upcoming issues of the MRS BULLETIN and in the mail.

Robin F.C. Farrow has worked in the field of molecular beam epitaxy (MBE) since 1968 and was one of the European pioneers of the technique. At the Royal Signals and Radar Establishment, Malvern, United Kingdom, he studied silicon epitaxy from molecular beams of silane and built prototype MBE machines for epitaxy of III-V compounds, including InP. During 1970 to 1980 he extended the MBE technique to new materials systems such as epitaxial insulators (alkaline earth fluorides), metastable phases (cubic tin) and lattice-matched III-VI/III-V interfaces (InSb/ CdTe). After a brief period at Westinghouse R\&D Center in Pittsburgh, Pennsylvania, he joined IBM in 1985 and is now at the IBM Almaden Research Center, San Jose, California, studying epitaxy of magnetic metals. In this work, ultrathin films of magnetic metals are prepared by MBE techniques and extensively characterized by a variety of in situ probes. The aim of this work is to understand the structure and defect dependence of magnetic prop- erties and to generate novel magnetic structures.

Richard $W$. Siegel received an MS in physics in 1960 and a PhD in metallurgy in 1965 from the University of Illinois in Urbana. From 1964 to 1966 he did postdoctoral research in the Materials Science and Engineering Department at Cornell University. Siegel served on the faculty of the State University of New York at Stony Brook in the Department of Materials Science from 1966 to 1976 . Since 1974 he has been a research scientist in the Materials Science Division at Argonne National Laboratory, first as a group leader in the areas of metal physics and defects in metals and presently as a research program manager. His present research interests primarily concern the synthesis, characterization, and properties of ultrafine-grained nanophase materials.

Siegel has been a visiting professor at research institutions in West Germany, Israel, and India, and is a member of several scientific societies, including the Materials Research Society. He has served on or chaired a variety of local, national, and international professional committees, and is an associate editor of the international journal, Materials Letters. He is the author or co-author of more than 80 publications. He was a co-organizer of the Symposium on the Characterization of Defects in Materials at the $1986 \mathrm{MRS}$ Fall Meeting, and is a co-organizer for the Symposium on Multicomponent Ultrafine Microstructures for the 1988 MRS Fall Meeting in Boston, November 28-December 3.

Angelica M. Stacy received her PhD in chemistry from Cornell University in 1981 and then did postdoctoral fellowship work at Northwestern University. In 1983 she joined the chemistry faculty at the University of California, Berkeley, where she is now an associate professor. Her current research group of 12 graduate students and two postdoctoral fellows has broad interests in solid state chemistry, with particular emphasis on the synthesis and characterization of new materials with novel elec-

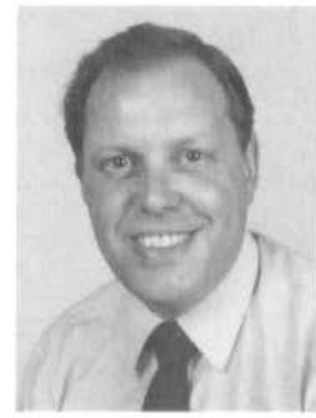

Robin Farrow

IBM Almaden Research Center (408) $927-2389$

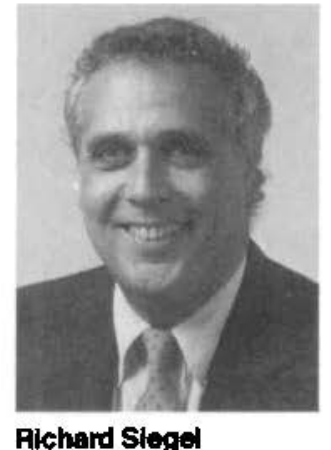

Argonne National Laboratory

(312) $972-4963$

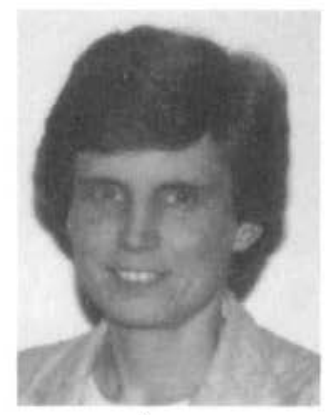

Angelica Stacy

Universtty of Calfiomia

(415) $642-3450$

tronic properties. Recent work includes the synthesis of: new ternary and quaternary copper oxides using molten hydroxides as solvents; low dimensional polychalcogenides by electrolysis; new ceramic phosphides by high temperature reactions; and high surface area transition metal nitrides and carbides. Stacy's group has discovered an unusual magnetic staircase in $\mathrm{EuNi}_{5} \mathrm{P}_{3}$, and a detailed investigation as to the origin of this behavior is under way. Her group is also investigating the surface chemistry of transition metal oxides under ultra-high vacuum conditions, and the role of the composition and structure of the interface between two reactants on the subsequent reaction. Stacy has received a Presidential Young Investigator Award from the $\mathrm{Na}$ tional Science Foundation and a Sloan Foundation Fellowship. 